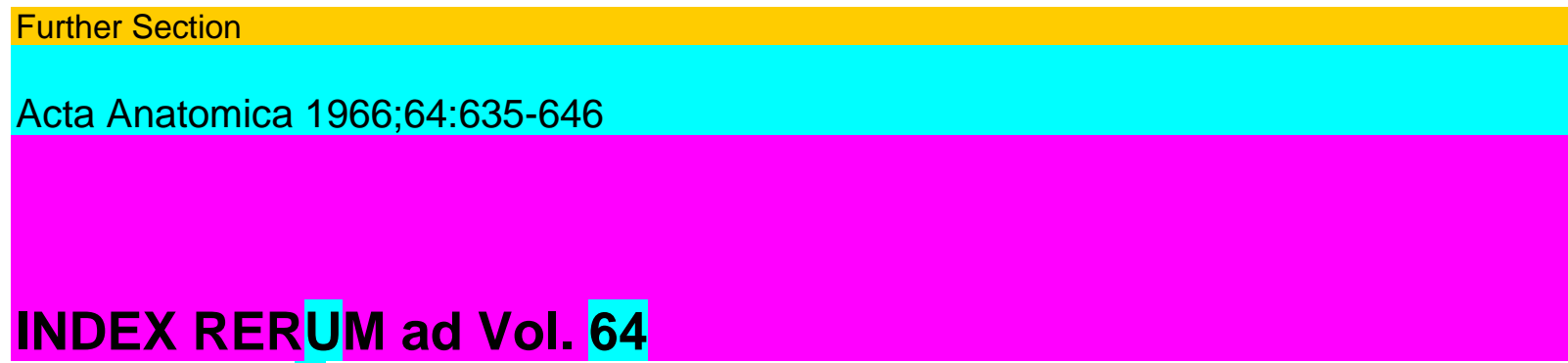

Confecit G. Boehm, Basel

Absorptive epithelium of the jejunum; the response of jejunal absorptive epithelium to an acute change in the internal environment of the gut . 295 Acetylcholinesterase, v. Stimmband

Actinopyga mauritania; calcified anal teeth and pharyngeal ring in the holothurian, Actinopyga mauritania 446

Adrenal cortex; influence of lesions of the cerebral cortex on the adrenal cortex

Adrenalectomy, v. Surrénalectomie

Ageing; morphological variations of the Golgi apparatus in spinal ganglion nerve cells, related to ageing 475

Albino mouse, v. Mouse

Albino rat, v. Rat

Alloxan diabetes; role of cortisone in the prevention of alloxan diabetic

cestrous cycle inhibition in rats $\quad 559$

Alter, v. Ageing

Ambystoma mexicanum; the influence of /?-aminopropionitrile on the carti lage matrix of the regenerating limbs of the axolotl (Ambystoma mexi

canum) (A histochemical study) 564

Ameloblasts; the function and maturity of ameloblasts and odontoblasts in relation to amelogenesis and dentinogenesis 351

Amelogenesis, v. Ameloblasts

Aminopropionitrile; the influence of /?-aminopropionitrile on the cartilage matrix of the regenerating limbs of the axolotl (Ambystoma mexicanum)

(A histochemical study) 564

Amnionstränge; über einen Fall von multiplen Extremitätenmißbildungen durch Amnionstränge 531

-; v. Foetal membranes

Anal teeth, calcified, and pharyngeal ring in the holothurian, Actinopyga mauritania 446

Anatomy, structural, of the inguinal region, contributions 179

Angio-architecture veineuse de la zone de transition æsophago-gastrique et son interpretation fonctionnelle

125

Anomalies, muscular, v. Muscular anomalies

Antelope; placenta and foetal membranes of the antelope, Ourebía ourebi

(Zimmermann)

390

Aorta; vergleichende metrische Untersuchungen am Endothel der Aorta und der Arteria pulmonalis des Kalbes

117

Apparatus digestorius, v. Enterochromaffin system 
Areola mammae, v. Peau aréolaire

Argentaffin cells, v. Intestine

Arm, v. Muscular anomalies

Arteria pulmonalis; vergleichende metrische Untersuchungen am Endothel

der Aorta und Arteria pulmonalis des Kalbes 117

Arterial segment 107

Autoradiographie; elektronenmikroskopische Autoradiographie, v. Knorpel-zelle

(B) $=$ Book reviews - Livres nouveaux - Buchbesprechungen

Acta anat., Vol. 64, No. 4 (1966) 48

636

Index rerum

Axolotl; the influence of /?-aminopropionitrile on the cartilage matrix of the regenerating limbs of the axolotl (Ambystoma mexicanum) (A histo-

chemical study) $\quad 564$

Balaenopteridae, v. Buckelwal

Baleine, v. Buckelwal

BAPN (=/?-Aminopropionitrile), v. Aminopropionitrile

Beta-aminopropionitrile (= BAPN), v. Aminopropionitrile

Bindegewebsapparat des menschlichen Stimmbandes; zur Frage der Endigung von Muskelfasern am Bindegewebsapparat des menschlichen

Stimmbandes 198

Biology; cell biology (4th ed.) (B) 634

Blood circulation, venous, v. Jejunal absorptive epithelium

Bone, v. Knochenbildung

Bourgeons musculaires, v. Skeletal muscle culture

Bovis, v. Endothel der Aorta

Brachium, v. Muscular anomalies

Brain and the lymphatic system, new contributions to their anatomical con nections 498

Brain, v. Cerebral cortex, Cerebrum

Bras, v. Muscular anomalies

Buckelwal; zum Hirnbau und Verhalten des Buckelwals, Megaptera novae-

anglíae, Borowski (Cetacea, Mysticeti, Balaenopteridae) 256

Bufo arenarum; contribution to the study of the epithalamus of Bufo are-

narum (Hensel) (I.: Histological observations on the epiphysis cerebri) 618

Bulbus caroticus, v. Carotid body

$\mathrm{CaC0} 3$ (calcite), v. Calcified anal teeth

Calcification, v. Ameloblasts

Calcified anal teeth and pharyngeal ring in the holothurian, Actinopyga Mau

ritania $\quad 446$

Calcified pharyngeal ring, v. Calcified anal teeth

Calf, v. Endothel der Aorta

Canalis alimentaríus, v. Intestine

Canaux épendymaires secondaires, v. Neuralrohr

Capillaries, v. Vasa vasorum

Carbon tetrachloride, v. Hepar 
Carotid body, an electron microscope study 163

Cartilage, v. Knorpelzelle

Cartilage matrix; the influence of $\wedge^{\wedge}$-aminopropionitrile on the cartilage ma trix of the regenerating limbs of the axolotl (Ambystoma mexicanum)

(A histochemical study) 564

Cat; variability of sensory nerve endings in foot pads of a domestic cat (Felis ocreata L., Felis domestica) 82

$-;$ v. Carotid body

Cavia porcellus, v. Cobaye, Cobaye gravide

CC14, v. Hepar

Cell biology (4th ed.) (B) 634

Cellules de Kultschitzky; examen du comportement des cellules de Kult-

schítzky après surrénalectomie unilatérale et bilatérale chez le cobaye 304

Cellules musculaires cardiaques, battant in vitro, v. Herzmuskelzellen

Cerebral cortex; influence of lesions of the cerebral cortex on the adrenal

cortex 1

Index rerum 637

Cerebrum; new contributions to the anatomical connections of the brain and

the lymphatic system 498

-; zum Hirnbau und Verhalten des Buckelwals, Megaptera novaeangliae

Borowski (Cetacea, Mystíceti, Balaenopterídae) 256

-; v. Epithalamus, Hypothalamus neurosécrétoire

Cerveau, v. Cerebral cortex, Cerebrum

Cetacea, v. Buckelwal

Chat, v. Carotid body, Cat

Cheek teeth; the disposition of the developing cheek teeth in the albino mouse 572

Chick embryo, v. Herzmuskelzellen, Skeletal muscle culture

Cholinesterase, v. Stimmband

Chondrocranium of the albino rat $\quad 586$

Chorda dorsalis, v. Notochord

Circulation veineuse, interromption, v. Jejunal absorptive epithelium

Cobaye; examen du comportement des cellules de Kultschitzky après sur-

rénalectomie unilatérale et bilatérale chez le cobaye 304

Cobaye gravide; la tétine et la peau aréolaire du cobaye gravide: Activité de

la glycéro-phosphatase alcaline

263

Cceur, v. Coronary arteries, Herzmuskelzellen (schlagende)

Cor, v. Coronary arteries, Herzmuskelzellen (schlagende)

Corde vocale inférieure, v. Stimmband, menschliches

Coronary arteries; an X-ray microscopic study of the postnatal development

of the vasa vasorum of normal human coronary arteries 506

Corpus pineale, v. Epiphysis cerebri

Cortex cerebri; influence of lesions of the cerebral cortex on the adrenal cortex

Cortex glandulae suprarenalis, v. Cortex cerebri

Cortisone, role in the prevention of alloxan diabetic oestrous cycle inhibition

in rats 559

Coslett-Níxon, X-ray projection microscope, v. Vasa vasorum 
Couronne calcaire péripharyngienne, v. Holothurian

Cranium, v. Chondrocranium

Crapaud, v. Bufo arenarum

Croissance des néoplasmes, action inhibitrice, v. Gewebsmastzelle

Culture des tissue, v. Herzmuskelzellen (schlagende), Skeletal muscle culture

Cytochemistry and cytology of the spermatogenesis of Manis crassicaudata,

the indian pangolin 462

Cytochemistry, v. Aminopropionitrile, Skeletal muscle culture, Stimmband,

Tétine Cytology and cytochemistry of the spermatogenesis of Manis crassicaudata,

the indian pangolin 462

Darm, v. Cellules de Kultschicky, Jejunal absorptive epithelium

«Dent anale», v. Holothurian

Dentes v. Cheek teeth, Dentinogenesis

Dentinogenesis; the function and maturity of ameloblasts and odontoblasts

in relation to amelogenesis and dentinogenesis

351

Development; the disposition of the developing cheek teeth in the albino

mouse 572

Development of vasa vasorum; an X-ray microscopic study of the postnatal

development of the vasa vasorum of normal human coronary arteries 506

Development, v. Ameloblasts, Cheek teeth, Chondrocranium, Muscular anomalies, Notochord,

Skeletal muscle culture

Diabetes, v. Alloxan diabetes

Difformities, v. Aminopropionitrile, Extremitätenmißbildungen, Muscular anomalies

638

Index rerum

Domestic cat, v. Cat

Dünndarm, v. Cellules de Kultschitzky, Jejunal absorptive epithelium

Eisen, Implantation, v. Knochenbildung

Elastiscbes Verhalten; Versuche zur Bestimmung des elastischen Verhaltens

des Uterus geschlechtsreifer Ratten unter dem Einfluß von Hyaluroni-

dase und während der Gravidität unter Berücksichtigung histologischer

Veränderungen $\quad 327$

Electron microscope study of the carotid body

163

Elektronenmikroskopisch-autoradiographische Untersuchung mit Tritium-markierter Glukose, v.

Knorpelzelle

Email, v. Ameloblasts

Embryo; the distribution of cells of the enterochromaffin system in the

gastrointestinal tract of human foetuses

-; sekundäre Lumenbildung im Neuralrohr und Rückenmark menschlicher

Embryonen 48

-; some muscular anomalies explained embryologically

-; v. Antelope, Cheek teeth, Chondrocranium, Extremitätenmißbildungen, Herzmuskelzellen,

Skeletal muscle culture

Enamelum, v. Ameloblasts

End plate, v. Endplatte, motorische

Endocrine organ of the intestine, v. Cellules de Kultschitzky 
Endocrinium, v. Hormones

Endothel der Aorta und Arteria pulmonalis des Kalbes, vergleichende metrische Untersuchungen 117

Endplatte, motorische; die Größenbeziehung von Muskelfaservolumen und Fläche der motorischen Endplatte bei verschiedenen Skeletmuskeln der Ratte $\quad 628$ Enterochromaffin system; the distribution of cells of the enterochromaffin system in the gastrointestinal tract of human foetuses $\quad 544$

-; v. Cellules de Kultschitzky

Entwicklung, v. Development

Epiphysis cerebri; contribution to the study of the epithalamus of Bufo arenarum (Hensel) (I.: Histological observations on the epiphysis cerebri) 618 Epithalamus; contribution to the study of the epithalamus of Bufo arenarum (Hensel) (I.: Histological observations on the epiphysis cerebri) . . 618 Epithelium, absorptive, of the jejunum; the response of jejunal absorptive epithelium to an acute change in the internal environment of the gut 295 Epithelium buccale, v. Cheek teeth

Ernährung, v. Germfree rat

Estomac, v. Gastrique

Estrus cycle, v. Oestrus cycle

Etapes de la vie, v. Golgi apparatus

Extremitätenmißbildungen; über einen Fall von multiplen Extremitäten mißbildungen durch Amnionstränge 531

Extremitates, v. Muscular anomalies, Regeneration of limbs

Feinstruktur, v. Carotid body, Knorpelzelle

Felis domestica, v. Carotid body, Felis ocreata

Felís ocreata; variability of sensory nerve endings in foot pads of a domestic cat (Felís ocreata L., Felís domestica) 82

Femur, v. Rattenfemur

Fer, implantation, v. Knochenbildung

Ferments, v. Hyaluronidase, Phosphatase alcaline, Stimmband, Succinic dehydrogenase

Index rerum 639

Fet..., v. Foet...

Fine structure, v. Carotid body, Knorpelzelle

Foetal membranes and placenta of the antelope Ourebia ourebi (Zimmermann) 390

-; v. Amnionstränge

Foetus, v. Embryo, Herzmuskelzellen, Skeletal muscle culture

Foetuses, human; the distribution of cells of the enterochromaf fin system in the gastrointestinal tract of human foetuses 544

Foie, v. Hepar

Fonctionnelle, interpretation fonctionnelle, v. Angio-architecture

Foot pads of a domestic cat (Felis ocreata L., Felis domestíca), variability of sensory nerve endings 82

Frakturbehandlung, v. Knochenbildung 
Frog tadpoles; role of the notochord in regeneration of the tail in frog tad

poles 341

Frosch, v. Frog tadpoles

Gallus domesticus, v. Herzmuskelzellen, Skeletal muscle culture

Gaster, v. Gastrique

Gastrique; sur Гangio-architecture de la zone de transition cesophago-

gastrique et son interpretation fonctionnelle 125

Gastrointestinal tract; the distribution of cells of the enterochromaf fin system

in the gastrointestinal tract of human foetuses

544

Gefäße, v. Angio-architecture, Arterial segment, Carotid body, Jejunal absorptive epithelium,

Vasa vasorum

Gehirn, v. Cerebral cortex, Cerebrum

Germfree rat, characteristics 367

Geschlechtsreife Ratten, v. Rattenuterus

Gestation, v. Gravidité

Gewebekultur, v. Herzmuskelzellen (schlagende), Skeletal muscle culture

Gewebsmastzelle; zur Frage der wachstumshemmenden Funktion der Ge-

websmastzelle 245

Glande mammaire, v. Tétine

Glande pinéale, v. Epiphysis cerebri

Glande thyroïde, v. Thyroidea

Glandulae suprarenales, v. Adrenal cortex, Surrénalectomie

Gliedmaßen, amputierte, v. Regeneration of limbs

Glomus caroticum, v. Carotid body

Glukose (H3-Glukose), v. Knorpelzelle

Glycéro-phosphatase alcaline, v. Gravidité

Golgi apparatus; morphological variations of the Golgi apparatus in spinal

ganglion nerve cells, related to ageing

475

Granula, metachromotrope, Ausstoßung, v. Gewebsmastzelle

Gravidité; la tétine et la peau aréolaire du cobaye gravide: Activate de la

glycéro-phosphatase alcaline 263

-; Versuche zur Bestimmung des elastischen Verhaltens des Uterus geschlechtsreifer Ratten unter dem Einfluß von Hyaluronidase und während der Gravidität unter Berücksichtigung histologischer Verände-

rungen 327

-; Antelope

Grenouille, v. Frog tadpoles

Grossesse, v. Antelope, Gravidité

Growth of tumours, inhibition, v. Gewebsmastzelle

Guinea-pig, v. Cobaye, Cobaye gravide

Gut; the response of jejunal absorptive epithelium to an acute change in

the internal environment of the gut 295

640

Index rerum

Haut des Warzenhofs (Meerschweinchen), v. Peau aréolaire

Heart, v. Coronary arteries 
Heart cells, isolated, pulsating in vitro, v. Herzmuskelzellen

Hepar; recent data concerning the connection between thyroid and hepatic

functions 235

Heparin, v. Gewebsmastzelle

Herz, v. Coronary arteries

Herzmuskelzellen (schlagende); Beitrag zur Technik der Gewinnung und

Züchtung in vitro schlagender Herzmuskelzellen 214

H3 (H3-Glukose), v. Knorpelzelle

Hirnbau und Verhalten des Buckelwals, Megaptera novaeangliae Borowski

(Cetacea, Mysticeti, Balaenopterídae) 256

Histochemistry, v. Aminopropionitrile, Skeletal muscle culture, Spermato-genesis, Stimmband,

Tétine

Holothurian; calcified anal teeth and pharyngeal ring in the holothurian,

Actinopyga maurítanía $\quad 446$

Hormones, v. Adrenal cortex, Enterochromaffin system, Oestrus cycle, Rattenuterus,

Rhinencéphale

Human coronary arteries; an X-ray microscopic study of the postnatal development of the vasa vasorum of normal human coronary arteries . 506

Human foetuses, v. Foetuses, human

Hyaluronidase; Versuche zur Bestimmung des elastischen Verhaltens des

Uterus geschlechtsreifer Ratten unter dem Einftuß von Hyaluronidase

und während der Gravidität unter Berücksichtigung histologischer

Veränderungen $\quad 327$

Hypothalamus neurosécrétoire; rhinencéphale et fonction structuraux de

Thypothalamus neurosécrétoire et du complexe endocrine après lesions

rhinencéphaliques

10

Implantation; vergleichende Untersuchungen über die reaktive Knochen-

bildung nach Implantation von Metallschrauben in den Rattenfemur 311

Incisive du rat, v. Ameloblasts

Indian pangolin, v. Pangolin, indian

Inguinal region; contribution to the structural anatomy of the inguinal region 179

Intestine; the distribution of cells of the enterochromaffin system in the

gastrointestinal tract of human foetuses $\quad 544$

-; v. Cellules de Kultschítzky, Germfree rat, Jejunal absorptive epithelium

Intoxication (CC14), v. Hepar

Iron, implantation, v. Knochenbildung

Isocortex cerebral, v. Cortex cerebri

Isotope, radioaktive, v. Knorpelzelle

Ivoire, v. Ameloblasts

Jejunal absorptive epithelium; the response of jejunal absorptive epithelium

to an acute change in the internal environment of the gut 295

Kalb; vergleichende metrische Untersuchungen am Endothel der Aorta und

der Arteria pulmonalis des Kalbes 117

Kalkkörper der Holothurien, v. Holothurian Kaninchen, v. Golgí apparatus Kapillaren, v. Vasa vasorum Karyometrie, v. Endothel der Aorta Katze, v. Carotid body, Cat Kaulquappen, v. Frog tadpoles Keimfreie Ratten, v. Germfree rat 


\section{Index reram}

641

Kernformindex (=Kernlänge/Kernbreite), v. Endothel der Aorta Knochenbildung; vergleichende Untersuchungen über die reaktive Knochen-

bildung nach Implantation von Metallschrauben in den Rattenfemur 311 Knorpel, Matrix, v. Aminopropionitrile Knorpelzelle; die Mucopolysaccharidsynthese in ihrer Beziehung zur submikroskopischen Struktur der Knorpelzelle (Elektronenmikroskopisch-

autoradiographische Untersuchung mit Tritium-markierter Glukose . 223 Kröte, v. Bufo arenarum Kultschitzky, cellules de -; examen du comportement des cellules de Kult-

schítzky après surrénalectomie unilatérale et bilatérale chez le cobaye . 304

Lapin, v. Golgi apparatus

Lathyrus odoratus ("lathyrus factor"), v. Aminopropionitrile

Lebensalter, v. Golgi apparatus

Leber, v. Hepar

Limbs, regeneration, v. Regeneration of limbs

Limbs, upper limbs, v. Muscular anomalies

Liver, v. Hepar

Lumenbildungen, sekundäre, im Neuralrohr und Rückenmark menschlicher

Embryonen $\quad 48$

Lymph stasis, experimental, v. Lymphatic system

Lymphatic system and brain, new contributions to their anatomical connec

tions 498

Magen, v. Gastriqpie

Male germ cells, v. Spermatogenesis

Malformations, v. Aminopropionitrile, Extremitätenmißbildungen, Muscular

anomalies Mamma, v. Tétine Man, v. Angio-architecture, Coronary arteries, Enterochromaffin

system,

Extremitätenmißbildungen, Inguinal region, Menschliche Embryonen,

Muscular anomalies, Stimmband Manis crassícaudata; cytology and cytochemistry of the spermatogenesis of

Manis crassicaudata, the indian pangolin $\quad 462$

Mastocytosis, v. Gewebsmastzelle

Mastzelle, Gewebsmastzelle; zur Frage der wachstumshemmenden Гunktion

der Gewebsmastzelle 245

Maus, v. Knorpelzelle, Mouse

Medulla spinalis, v. Rückenmark

Meerschweinchen, v. Cobaye, Cobaye gravide

Megaptera novaeangliae; zum Hirnbau und Verhalten des Buckelwals, Mega-

ptera novaeangliae Borowski (Cetacea, Mysticeti, Balaenopteridae) . . 256 Membres, v.

Extremitätenmißbildungen, Muscular anomalies Menschliche Embryonen; sekundäre

Lumenbildungen im Neuralrohr und

Rückenmark menschlicher Embryonen

48

Menschliches Stimmband; zur Frage der Endigung von Muskelfasern am

Bindegewebsapparat des menschlichen Stimmbandes 198

Metachromotrope Granula, Ausstoßung, v. Gewebsmastzelle

Metall (Metallschrauben), Implantation, v. Knochenbildung 
Methode, v. Herzmuskelzellen (schlagende)

Metrische Untersuchungen, vergleichende, am Endothel der Aorta und Arteria pulmonalis des Kalbes 117

Mice, v. Knorpelzelle, Mouse

Microautoradiographie; elektronenmikroskopische Autoradiographie, v.

Knorpelzelle

642

Index rerum

Microscope électronique, v. Carotid body, Knorpelzelle

Microscope, X-ray projection microscope (Coslett-Nixon), v. Vasa vasorum

Mißbildungen, v. Aminoproprionitrile, Extremitätenmißbildungen, Muscular anomalies

Moelle épinière, v. Medulla spinalis

Molaires, v. Cheek teeth

Motorische Endplatte; die Größenbeziehung von Muskelfaservolumen und

Fläche der motorischen Endplatte bei verschiedenen Skeletmuskeln der

Ratte 628

Mouse; the disposition of the developing cheek teeth in the albino mouse . 572

Mucopolysaccharidsynthese in ihrer Beziehung zur submikroskopischen

Struktur der Knorpelzelle (Elektronenmikroskopisch-autoradiographi-

sche Untersuchung mit Tritium-markierter Glukose) 223

Mucosa, v. Angio-architecture, Jejunal absorptive epithelium

Multiple Extremitätenmißbildungen; über einen Fall von multiplen Extre

mitätenmißbildungen durch Amnionstränge 531

Mus musculus, v. Knorpelzelle, Mouse

Muscle buds, v. Muscle, skeletal

Muscle culture, v. Muscle, skeletal

Muscle, skeletal; location of succinic dehydrogenase in skeletal muscle cul ture of chick embryos 282

Muscular anomalies; some unusual muscular anomalies explained embryo-

logically 517

Musculus thyreoarytaenoideus; zur Frage der Endigung von Muskelfasern

am Bindegewebsapparat des menschlichen Stimmbandes 198

Muskel, glatter (Uterus), v. Elastisches Verhalten

Muskelfasern, Endigung, v. Musculus thyreoarytaenoideus

Muskelfaservolumen; die Größenbeziehung von Muskelfaservolumen und

Fläche der motorischen Endplatte bei verschiedenen Skeletmuskeln

der Ratte $\quad 628$

Muskelzellen, v. Herzmuskelzellen (schlagende)

Myoneural junction, v. Endplatte, motorische

Mystíceti, v. Buckelwal

Nebennieren, v. Surrénalectomie

Nebennierenrinde, v. Adrenal cortex

Nerve cells; morphological variations of the Golgi apparatus in spinal gan

glion nerve cells, related to ageing $\quad 475$

Nerve endings, sensory; variability of sensory nerve endings in foot pads of

a domestic cat (Felís ocreata L., Felís domestica) 
Nerven (Nervensystem, Zentralnervensystem), v. Buckelwal, Carotid body, Cerebral cortex, Endplatte, Epithalamus, Lymphatic system, Nerve cells, Nerve endings, Neurosécrétion Neuralrohr und Rückenmark menschlicher Embryonen, sekundäre Lumenbildungen $\quad 48$

Neurosécrétion; rhinencéphale et fonction neuroendocrine: Aspects structuraux de l'hypothalamus neurosécrétoire et du complexe endocrine après lesions rhinencéphaliques $\quad 10$

Nipple, v. Tétine

Nixon, v. Coslett-Nixon...

Nomarthra, v. Manis

Notochord, role in regeneration of the tail in frog tadpoles 341

Nourriture, v. Germfree rat

Noyau, forme (= longueur nucléaire/largeur nucléaire), v. Endothel der Aorta

Index rerum 643

Nuclear-form-index (= nuclear length/nuclear width), v. Endothel der Aorta Nutrition, v.

Germfree rat

Odontoblasts; the function and maturity of ameloblasts and odontoblasts

in relation to amelogenesis and dentinogenesis 351

Oesophagus; sur l'angio-architecture veineuse de la zone de transition oeso-

phago-gastrique et son interpretation fonctionnelle 125

Oestrous cycle; role of cortisone in the prevention of alloxan diabetic oestrous

cycle inhibition in rats 559

Ontogenesis, v. Antelope, Cheek teeth, Chondrocranium, Enterochromaffin system,

Extremitätenmißbildungen, Menschliche Embryonen, Muscular anomalies, Notochord, Skeletal

muscle culture

Oral epidermis, v. Cheek teeth

Oryctolagus cunículus, v. Golgi apparatus

Os, v. Knochenbildung

Ourebía ourebi; placenta and foetal membranes of the antelope, Ourebia ou-

rebí (Zimmermann) 390

Pads, foot pads, v. Sensory nerve endings

Pangolin, indian; cytology and cytochemistry of the spermatogenesis of

Manis crassicaudata, the indian pangolin 462

Papilla mammae, v. Tétine

Patte amputee, v. Regeneration of limbs

Patte du chat, v. Sensory nerve endings

Peau aréolaire et tétine du cobaye gravide: Activité de la glycéro-phosphatase

alcaline $\quad 263$

Pelotes digitales et métacarpiennes, v. Sensory nerve endings

Pfoten der Katze, v. Sensory nerve endings

Pharyngeal ring and calcified anal teeth in the holothurian, Actinopyga

tnauritanía 446

Pholídita, v. Manis

Phosphatase alcaline; la tétine et la peau aréolaire du cobaye gravide: Acti

vité de la glycéro-phosphatase alcaline

263

Pineal organ, v. Epipbysis cerebri 
Placenta and foetal membranes of the antelope, Ourebía ourebi (Zimmer mann) 390

-; v. Amnionstränge

Plaque motrice, v. Endplatte, motorische

Plicae vocales, v. Stimmband

Postnatal development, v. Vasa vasorum

Poulet (embryon), v. Herzmuskelzellen, Skeletal muscle culture

Pregnancy, v. Antelope, Gravidität

Pulmonary artery, endothelium, v. Endothel der Aorta

Pulsierende isolierte Herzmuskelzellen; Beitrag zur Technik der Gewinnung und Züchtung ire vitro schlagender Herzmuskelzellen 214

Queue de têtards, v. Regeneration

Rabbit, v. Golgi apparatus

Rana clamítans, v. Frog tadpoles

Rat; the chondrocranium of the albino rat

586

-; die Größenbeziehung von Muskelfaservolumen und Fläche der motori-

schen Endplatte bei verschiedenen Skeletmuskeln der Ratte .... 628

-; role of cortisone in the prevention of alloxan diabetic oestrus cycle in

hibition in rats 559

-; v. Gewebsmastzelle, Hepar, Jejunal absorptive epithelium

644

Index rerum

Rat, germfree, characteristics 367

Rat incisors, v. Ameloblasts

Rattenfemur; vergleichende Untersuchungen über die reaktive Knochen-

bildung nach Implantation von Metallschrauben in den Rattenfemur . 311

Rattenuterus; Versuche zur Bestimmung des elastischen Verhaltens des

Uterus geschlechtsreifer Ratten unter dem Einfluß von Hyaluronidase

und während der Gravidität unter Berücksichtigung histologischer

Veränderungen 327

Rayons X, v. X-ray microscopic...

Reaktive Knochenbildung; vergleichende Untersuchungen über die reaktive

Knochenbildung nach Implantation von Metallschrauben in den Rat

tenfemur $\quad 311$

Receptors, v. Sensory nerve endings

Regeneration; role of the notochord in regeneration of the tail in frog tad poles 341

Regeneration of limbs; the influence of $\wedge^{\wedge}$-aminopropionitrile on the cartilage matrix of the regenerating limbs of the axolotl (Ambystoma mexicanum),

(A histochemical study) 564

Regio inguinalis, $v$. Inguinal region

Rhinencéphale et fonction neuroendocrine: Aspects structuraux de l'hypo-

thalamus neurosécrétoire et du complexe endocrine après lesions rhin-

encéphaliques 10

Röntgenstrahlen, v. X-ray microscopic.

Rückenmark und Neuralrohr menschlicher Embryonen, sekundäre Lumen- 
bildungen 48

Schädel, v. Chondrocranium

Schilddrüse, v. Thyreoidea

Schlagende isolierte Herzmuskelzellen; Beitrag zur Technik der Gewinnung und Züchtung in vitro schlagender Herzmuskelzellen 214

Schleimhaut, v. Angio-architecture, Jejunal absorptive epithelium

Schuppentier, v. Manis

Schwangerschaft, v. Antelope, Gravidité

Schwanz von Kaulquappen, v. Regeneration

Schwellmechanismus der Schleimhaut der Übergangszone von Oesophagus

zu Magen, v. Angio-architecture

Segment, arterial 107

Sekundäre Lumenbildungen im Neuralrohr und Rückenmark menschlicher

Embryonen $\quad 48$

Sensory nerve endings; variability of sensory nerve endings in foot pads of

a domestic cat (Felis ocreata L., Felis domestica) 82

Sex organs, v. Rattenuterus, Spermatogenesis

Sinus caroticus, v. Carotid body

Skeletal muscle culture of chick embryos, location of succinic dehydrogenase 282

Skeletmuskeln; die Größenbeziehung von Muskelfaservolumen und Fläche

der motorischen Endplatte bei verschiedenen Skeletmuskeln der Ratte 628 Skin of the Areola

mammae (guinea-pig), v. Peau aréolaire Skull, v. Chondrocranium

Smooth muscle (uterus), v. Elastisches Verhalten Souris, v. Knorpelzelle, Mouse

Spermatogenesis; cytology and cytochemistry of the spermatogenesis of Ma

nis crassicaudata, the indian pangolin 462

Spinal cord, v. Medulla spinalis

Spinal ganglion; morphological variations of the Golgi apparatus in spinal

ganglion nerve cells, related to ageing $\quad 475$

Index rerum

645

Squamata, v. Manis

Stahl (V 4 A-Stahl), Implantation, v. Knochenbildung

Statistische Auswertung, v. Endothel der Aorta, (Golgi apparatus)

Steroide, v. Cortisone

Stimmband, menschliches; zur Frage der Endigung von Muskelfasern am

Bindegewebsapparat des menschlichen Stimmbandes 198

Stomach, v. Gastrique

Structural anatomy of the inguinal region, contributions

Submicroscopical structure, v. Carotid body, Knorpelzelle

Succinic dehydrogenase; location of succinic dehydrogenase in skeletal

muscle culture of chick embryo 282

Surrénalectomie; examen du comportement des cellules de Kultschítzky après

surrénalectomie unilatérale et bilatérale chez le cobaye $\quad 304$

Surrénales, v. Adrenal cortex

«Syntacoben» (= Metallegierung), Implantation, v. Knochenbildung

Systema digestorium, v. Intestine 
Tadpoles; role of the notochord in regeneration of the tail in frog tadpoles . 341

Tail in frog tadpoles, regeneration, role of the notochord

341

Technik, v. Herzmuskelzellen (schlagende)

Teeth, v. Cheek teeth, Dentinogenesis

Teeth, anal, calcified and pharyngeal ring in the holothurian, Actinopyga

tnauritania 446

Teratogenic effect, v. Aminopropionitrile

Teratologie, v. Extremitätenmißbildungen, Muscular anomalies

Terminaisons nerveuses sensibles, v. Sensory nerve endings

Testes, v. Spermatogenesis

Têtards, v. Frog tadpoles

Tétine et la peau aréolaire du cobaye gravide: Activité de la glycéro-phos-

phatase alcaline 263

Tetrachlorkohlenstoff (Vergiftung), v. Hepar

«Thybon» (Hoechst), v. Thyreoidea

Thyreoidea; recent data concerning the connection between thyroid and

hepatic functions 235

Tissue cultures, v. Herzmuskelzellen (schlagende), Skeletal muscle culture

Toad, v. Bufo arenarum

Tooth, v. Cheek teeth, Dentinogenesis

Tooth-like calcified structures, v. Holothurian

Topographic anatomy, v. Inguinal region

Trächtigkeit, v. Antelope, Gravidité

Transition; oesophago-gastric transition, v. Angio-architecture

L-3,5,3'-Trijodothyronin (= «Thybon», Hoechst), v. Thyreoidea

Tritium-markierte Glukose, v. Knorpelzelle

Tumoren, Wachstumshemmung; zur Frage der wachstumshemmenden

Funktion der Gewebsmastzelle 245

Tunica mucosa, v. Angio-architecture, Cellules de Kultschítzky, Jejunal absorptive epithelium

Turgescence de la muqueuse oesophago-gastrique (zone de transition), v. Angio - architecture

Übergangszone von Oesophagus zu Magen, v. Angio-architecture

Ultrastructure, v. Carotid body, Knorpelzelle

Uterus; Versuche zur Bestimmung des elastischen Verhaltens des Uterus

geschlechtsreifer Ratten unter dem Einfluß von Hyaluronidase und

während der Gravidität unter Berücksichtigung histologischer Ver-

änderungen 327

646

Index rerum

V 4 A-Stahl, Implantation, v. Knochenbildung

Vaisseaux, v. Arterial segment, Carotid body, Jejunal absorptive epithelium,

Vasa vasorum, Veines Variability of sensory nerve endings in foot pads of a domestic cat (Felis

ocreata L., Felis domestíca) 82

Vasa vasorum; an X-ray microscopic study of the postnatal development of

the vasa vasorum of normal human coronary arteries $\quad 506$

Veau, v. Endothel der Aorta

Veines; sur l'angio-architecture veineuse de la zone de transition æsophago- 
gastrique et son interpretation fonctionnelle 125

Venous blood circulation, blockade, v. Jejunal absorptive epithebum

Vergiftung (Tetracblorkohlenstoff), v. Hepar

Vergleichende metrische Untersuchungen am Endothel der Aorta und der

Arteria pulmonalis des Kalbes

117

Verhaltensforschung, v. Buckel·wal

Vessels, v. Arterial segment, Carotid body, Jejunal absorptive epithelium,

Vasa vasorum, Veines Vital cycle, v. Golgi apparatus Vocal cord, v. Stimmband

Wachstumshemmung; zur Frage der wachstumshemmenden Funktion der

Gewebsmastzelle

245

Wal, v. Buckelwal

Warzenhof (Meerscbweinchen), v. Peau aréolaire

Whale, v. Buckelwal

X-ray microscopic study of the postnatal development of the vasa vasorum

of normal human coronary arteries 506

Zähne, v. Cheek teeth, Dentinogenesis

Zahnähnliche (verkalkte) Gebilde, v. Holothurian

Zellkultur, v. Herzmuskelzellen (schlagende), Skeletal muscle culture

Zitze, v. Tétine

Zone de transition cesophago-gastrique, v. Angio-architecture

New books - Livres nouveaux - Buchbesprechungen

de Robertis, E.D. P., Nowínski, W. W. and Saetz, F.A.: Cell biology (4th ed.)

(W.B.Saunders, Philadelphia/London 1965) (Bef.: O.Bucher) ... 634 